\title{
Symposium
}

\section{Beiträge zu Claus Offes Arbeitsmarktsoziologie}

Claus Offe, Macht und Effizienz. Studien zur kapitalistischen Rationalisierung der Arbeit, Wiesbaden: Springer VS (Ausgwählte Schriften von Claus Offe Band 1), 2018. 384 S., gb., 64,99€

Claus Offe, Der Wohlfahrtsstaat und seine Bürger, Wiesbaden: Springer VS (Ausgewählte Schriften von Claus Offe Band 2), Wiesbaden: Springer VS, 2019, 336 S., gb., 64,99€

Claus Offe, Institutionen, Normen, Bürgertugenden, Wiesbaden: Springer VS (Ausgewählte Schriften von Claus Offe Band 3), 2019, 434 S., gb., 69,99€

Claus Offe, Liberale Demokratie und soziale Macht. Demokratietheoretische Studien, Wiesbaden: Springer VS (Ausgewählte Schriften von Claus Offe Band 4), 2019, 388 S., gb., 59,99€

Claus Offe, Staatskapazität und Europäische Integration, Wiesbaden: Springer VS (Ausgewählte Schriften von Claus Offe Band 5), 2019, 395 S., gb., 64,99€

Claus Offe, Übergänge. Vom Staatssozialismus zum demokratischen Kapitalismus, Wiesbaden: Springer VS (Ausgewählte Schriften von Claus Offe Band 6), 2020, 388 S., gb., 59,99€

Besprochen von Prof. Dr. Martin Brussig: Institut Arbeit und Qualifikation (IAQ), Universität Duisburg-Essen, E-Mail: martin.brussig@uni-due.de

https://doi.org/10.1515/srsr-2021-0046

Schlüsselwörter: Arbeitsmarktsoziologie, Theorie des Arbeitsmarktes, Geschichte der Soziologie

1.

Gleich zu den Beiträgen von Claus Offe zur Soziologie von Arbeitsmärkten, vorab nur: Die soziologische Arbeitsmarktforschung hat sich in der Bundesrepublik Deutschland erst in den 1970er Jahren als ein eigenständiges Gebiet der Soziologie herausgebildet. Seitdem ist sie in vielfältige Richtungen gewachsen, und hat mehrspurige, ungerichtete, suchende, wechselvolle Entwicklungen genommen statt - etwa auf der Grundlage eines hegemonialen Forschungsprogramms - ei- 
nen klaren Kurs zu ziehen. Dies zeigt sich nicht zuletzt an zentralen Indikatoren für die Etablierung einer Subdisziplin: Der in den 1970er Jahren gegründete und noch heute bestehende Arbeitskreis „Sozialwissenschaftliche Arbeitsmarktforschung“ (SAMF) betont schon in seinem Namen die Interdisziplinarität des Feldes, ohne sich im engen Sinn der Soziologie zuzurechnen; und ebenso hat es die lange Zeit wichtigste Fachzeitschrift für Arbeitsmarktsoziologie gehalten, die „Mitteilungen aus der Arbeitsmarkt- und Berufsforschung“, und zwar selbst über den Namenswechsel hin zum heutigen „Journal for Labour Market Research“. Es gibt bis heute keine Sektion „Arbeitsmarktsoziologie“ innerhalb der Deutschen Gesellschaft für Soziologie (DGS). Die Entwicklung der soziologischen Arbeitsmarktforschung ist von Beginn an durch den Austausch mit anderen sozialwissenschaftlichen Disziplinen geprägt, und dies vielleicht in einer noch stärkeren Weise, als benachbarte soziologische Gebiete bzw. Subdisziplinen mit ebenfalls unscharfen Grenzen, wie etwa die Arbeits- und Industriesoziologie (die von Anfang an mehr war als eine Soziologie der Industrie), die Organisationssoziologie oder die Wirtschaftssoziologie.

Trotz dieser unvollständigen Ausdifferenzierung als spezifisch soziologische Teildisziplin ist eine lebendige soziologische Arbeitsmarktforschung entstanden. Mit erheblicher, aber hoffentlich zulässiger Vergröberung lässt sich mit dem Wechsel der Jahrzehnte eine Abfolge dominanter Themen und Fragestellungen beschreiben: In den 1970er Jahren waren die frühen segmentationstheoretischen Studien von Burkart Lutz und Werner Sengenberger ein wichtiger Ausgangspunkt. Deren Grundideen wurden teilweise aus der US-amerikanischen Arbeitsmarktforschung importiert, dann aber an die institutionellen Besonderheiten der Bundesrepublik - namentlich der Sozialfigur des Facharbeiters - angepasst. Dies verband sich mit einer besonderen Aufmerksamkeit für den Betrieb als strukturprägende Kraft auf dem Arbeitsmarkt. In den 1980er Jahren rückten Fragen des Strukturwandels zur Dienstleistungsökonomie in den Vordergrund, verbunden mit Fragen zur Gestaltung von Arbeitszeit (besonders prominent zur Gestaltung der Wochen- und Lebensarbeitszeit), wie sie etwa von Karl Hinrichs, Gerhard Bosch, Ulrike Berger und anderen bearbeitet wurden. Dies war auch gesellschaftspolitisch motiviert, denn die bestehende Massenarbeitslosigkeit von damals skandalösen Ausmaßen schien gegen alle Versuche politischer Einflussnahme resistent.

In den 1990er Jahren verschob sich der Fokus der soziologischen Arbeitsmarktforschung, indem auf die enge Verbindung der Arbeitsmarkt- mit der Industrie- und Betriebssoziologie nun eine stärkere Verknüpfung mit der Sozialstrukturanalyse folgte. Wobei „folgen“ nicht das richtige Wort ist, denn es gab weiterhin Untersuchungen zu einer betriebsbezogenen Arbeitsmarktsoziologie. Aber der Fokus verschob sich. Fragen zur individuellen Mobilität auf Arbeitsmärkten und zur Stabilität von Beschäftigungsverhältnissen sowie Untersuchungen zur 
Neustrukturierung des Arbeitsmarktes in Ostdeutschland regten zu einer Vielzahl von Studien auf der Grundlage neu verfügbarer Daten an. Matthias Knuth und Marcel Erlinghagen gehörten zu den ersten, die mit der IAB-Beschäftigtenstichprobe gearbeitet und die mit ihren Interpretationen gängige Deutungen zum Arbeitsmarktgeschehen in Frage gestellt haben. Die Arbeit mit Daten, die durch Aktivitäten von Sozialleistungsträgern anfallen, war damals noch neu gegenüber Daten, die auf Befragungen beruhen und auf denen frühere Mobilitätsstudien beruhten, und sie gewann rapide an Bedeutung.

Teilweise darauf aufbauend und teilweise wiederum neu ansetzend, verschob sich der Fokus seit Ende der 1990er Jahre abermals und richtete sich nun verstärkt auf Fragen der Arbeitsmarktpolitik, womit aber nicht sämtliche politischen Fragen gemeint sind, die im Zusammenhang mit Arbeitsmärkten behandelt werden. Gemeint ist ein eigentlich recht schmaler Ausschnitt, der mit „Arbeitsförderung“ treffender benannt ist und arbeitsmarktpolitische Fragen im Zusammenhang mit dem Auftreten von Arbeitslosigkeit und der Frage, wie Arbeitslose wieder in Beschäftigung kommen, untersucht. Vielleicht erstmals in der Geschichte der Arbeitsmarktsoziologie der Bundesrepublik erfolgte nun auf breiter Front ein Anschluss an internationale Diskurse, denn unter dem Stichwort der ,aktivierenden Arbeitsmarktpolitik“ fand ein Wandel der Arbeitsmarktpolitik in vielen entwickelten Ländern insbesondere in Europa statt, der länderspezifisch ausfiel, sich aber einer gemeinsamen Begrifflichkeit bediente. Beispielhaft für diese Debatten ist Günther Schmid zu nennen. Über die gesamte Zeit hinweg und bis in die Gegenwart hinein hat die Arbeitsmarktforschung ihre empirische Basis ausgebaut, zudem hat die Beratung von Politik und Verwaltung an Gewicht gewonnen.

Inzwischen besteht ein Konsens darin, dass sich das „Aktivierungsparadigma“ politisch erschöpft und als wissenschaftliche Inspiration an Glanz verloren hat. Was an seine Stelle tritt, ist, wie immer, wenn es um die Gegenwart geht, schwer zu sagen. Ist es die neuerliche Befassung mit sozialstrukturellen Fragen in Verbindung mit einer Neuauflage der Segmentationstheorie unter dem Stichwort der „Dualisierung“, wie es angesichts der Diskussionen um soziale Ungleichheit mitunter scheint? Oder zieht die Arbeitsmarktsoziologie eher aus den Herausforderungen aufgrund der Digitalisierung die Kraft für aktuelle Impulse?

\section{II.}

Claus Offe gehört zu den Wenigen, die nicht nur über nahezu den gesamten hier skizzierten Zeitraum zur Arbeitsmarktsoziologie beigetragen haben, sondern darüber hinaus zu den sehr Wenigen, die in der Arbeitsmarktsoziologie in ihrer vollen Breite gewirkt haben. Und das, so ist hinzuzufügen, aus der relativ randstän- 
digen Position eines Lehrstuhlinhabers mit diversen Forschungsaufenthalten in aller Welt, aber ohne die Ressourcen, die sich aus der Leitung eines Instituts oder eines Fachverbandes ergeben können. In der sechsbändigen Werkausgabe ausgewählter Aufsätze konzentrieren sich die Aufsätze zu Fragen des Arbeitsmarktes in Band 1 (Macht und Effizienz. Studien zur kapitalistischen Rationalisierung der Arbeit), ohne darauf beschränkt zu sein. Zugleich ist dies der Band mit den meisten Beiträgen in Ko-Autorenschaft und es handelt sich um - im Durchschnitt eher ältere Aufsätze, wenngleich die Aufsätze auf die Bände nicht chronologisch verteilt sind. Insgesamt wird man aber sagen können, dass sich das Forschungsinteresse von Claus Offe im Laufe der Zeit von Fragen des Arbeitsmarktes eher abund anderen Fragen zugewandt hat.

Der Aufsatz „Sozialökonomie des Arbeitsmarktes: primäres und sekundäres Machtgefälle“ (orig. 1984, mit Karl Hinrichs; hier abgedruckt in Band 1: 3-42) enthält eine klassische Grundlegung der Arbeitsmarktsoziologie. ${ }^{1}$ Er beinhaltet zum einen eine Bestimmung der Besonderheiten des Arbeitsmarktes gegenüber anderen Märkten. Diese Besonderheit resultiert wesentlich aus der Tatsache, dass bei einem Preisverfall (Lohnrückgang) kein Angebotsrückgang zu erwarten ist. Dadurch bildet sich eine grundlegende Machtasymmetrie zugunsten der Nachfrageseite (Arbeitgeber) gegenüber der Angebotsseite (Beschäftigte und Arbeitsuchende) am Arbeitsmarkt (,primäres Machtgefälle“). Zum zweiten werden Besonderheiten des Arbeitsvertrages gegenüber anderen Verträgen erläutert. Hierzu gehört die Tatsache, dass die Aufgaben im Arbeitsvertrag nicht im Vorhinein, also mit Vertragsabschluss, ausreichend genau spezifiziert werden. Daraus ergibt sich eine charakteristische Unbestimmtheit des Arbeitsvertrages, aus der wiederum wichtige Konsequenzen für die Arbeitsorganisation folgen, die unter dem Stichwort des „Kontrollproblems“ diskutiert werden. Zum dritten wird der Arbeitsmarkt im Verhältnis zu anderen Organisationsformen menschlicher Arbeitskraft dargestellt. Der zentrale Punkt ist hier, dass die Grenzen dessen, was marktlichvertraglich geregelt ist, gesellschaftlich bzw. staatlich gezogen sind. Aus diesen Grenzziehungen heraus entstehen vier Gruppen. Offe unterscheidet abhängig Beschäftigte, Selbstständige, Beschäftigungssuchende und „Inaktive“. Aus der Differenzierung in diese vier Gruppen - und aus den Strategien von Arbeitgebern, Beschäftigten und Staat zur Regulierung des „primären Machtgefälles“ - resul-

\footnotetext{
1 Eine - in den vorliegenden Bänden nicht abgedruckte - Vorfassung wurde 1977 veröffentlicht (Offe, C.; Hinrichs, K. (1977): Sozialökonomie des Arbeitsmarktes und die Lage „benachteiligter“ Gruppen von Arbeitnehmern. In: Opfer des Arbeitsmarktes. Zur Theorie der strukturierten Arbeitslosigkeit. 1. Aufl. Offe, C., Hrsg.; Neuwied, Darmstadt: Luchterhand (Kritische Texte: Sozialarbeit, Sozialpädagogik, soziale Probleme), pp 3-61.
} 
tiert eine weitere Machtasymmetrie innerhalb der Angebotsseite, also der Beschäftigten bzw. potenziell Beschäftigten („sekundäres Machtgefälle“).

Der Frage, durch welche Mechanismen primäres und sekundäres Machtgefälle ihre gesellschaftlich konkrete Ausprägung erhalten, sind weitere wegweisende Aufsätze gewidmet, von denen hier zwei zu nennen sind: „Two Logics of Collective Action“ (orig. 1980, mit Helmut Wiesenthal; hier abgedruckt in Band 1: 315-362) behandelt Fragen zur Organisation von Interessen auf der Angebots- und Nachfrageseite auf dem Arbeitsmarkt, und der Aufsatz „Politisch-soziologische Erklärungsansätze für Funktionen und Innovationsprozesse der Sozialpolitik“ (orig. 1977, mit Gero Lenhardt; hier abgedruckt in Band 2: 3-38) behandelt die Rolle des Staates. „Two Logics“ knüpft an die Feststellung von Machtasymmetrien an und entwickelt den Grundgedanken, dass Gewerkschaften sowohl eine monologische vor allem eine auf externe Umwelten bezogene - als auch eine dialogische - auf das Organisationsinnere bezogene - Logik kollektiven Handelns nutzen müssen. Für deren unmittelbare Kontrahenten, die Arbeitgeberverbände, wird hingegen konstatiert, dass sie sich sehr viel stärker auf eine monologische Logik beschränken können. Dadurch gewinnen Arbeitgeberverbände erhebliche Effizienzvorteile in der Machtartikulation und Interessendurchsetzung. Für den Erfolg von Gewerkschaften ist zudem deren Akzeptanz durch das politische System erforderlich. Dies stärkt die monologische Logik, die dazu beiträgt, dass Forderungen der Gewerkschaften bei denen Gehör finden, die die Gewerkschaften als Vertragspartner adressieren. Zugleich schwächt die monologische Logik die Mitgliedermobilisierung, denn letztere beruht auf der dialogischen Logik. Offe und Wiesenthal entwickeln ein zyklisches Phasenmodell gewerkschaftlichen Handelns, das bei der Selbstorganisation beginnt und über mehrere Schritte nach deren Konstitutionalisierung wiederum zu einer Re-Politisierung von Gewerkschaften führt.

In der Sozialpolitik - der Akteur ist hier nicht so richtig klar - gibt es ebenfalls Spannungsverhältnisse, die im Arbeitsmarkt ihren Ausgangspunkt haben und die Machtverhältnisse auf Arbeitsmärkten prägen. Offe und Lenhardt konstatieren, „Sozialpolitik ist die staatliche Bearbeitung des Problems der dauerhaften Transformation von Nicht-Lohnarbeitern in Lohnarbeiter“ (zit. nach Band 2: 8). Sozialpolitik dient also nicht nur der Kompensation von Arbeitsmarktrisiken, sondern ebenso gewissermaßen der Herstellung von Arbeitsmärkten, und hierfür sind drei Probleme zu lösen: „das Problem der Eingliederung der Arbeitskräfte in die Angebotsseite des Arbeitsmarktes, die Institutionalisierung der im Lohnarbeitsverhältnis nicht ,unterzubringenden“ Lebenssphären und Lebensrisiken und der quantitativen Regulierung des Verhältnisses zwischen Angebot und Nachfrage auf dem Arbeitsmarkt“ (ebda.: 15). Damit werden Lebensweisen jenseits einer Integration auf Arbeitsmärkten strukturell erschwert und ideologisch delegitimiert. Aus dieser Perspektive sind Lohnarbeitsverhältnisse zweischneidig, da sie nicht 
nur individuelle Verwirklichungschancen bieten, sondern auch Unterwerfung einfordern. Viele der an dieser Stelle von Offe abgeleiteten - empirisch untersucht sind sie nicht - Entwicklungstendenzen der Arbeitsmarktpolitik und des Sozialstaates muten erstaunlich modern an, so etwa der Übergang von Final- zu Konditionalprogrammen, eine Betonung präventiver Sozialpolitik, ein wachsender Stellenwert von Dienstleistungen gegenüber Geldleistungen und nicht zuletzt eine Verwissenschaftlichung von Sozialpolitik (ebda.: 27-36).

Diese drei beispielhaft herausgegriffenen Aufsätze fallen in die Gründungsphase der Arbeitsmarktsoziologie und bieten ihr ein theoretisches Fundament. Die dort entwickelten Überlegungen ziehen sich durch eine Reihe von Aufsätzen und benennen den Kern dessen, was in der Perspektive von Claus Offe den Arbeitsmarkt aus einer soziologischen Perspektive ausmacht. Eine wichtige Konsequenz ist, dass sogenannte „Randgruppen des Arbeitsmarktes“ - und Offe benutzt in einem Beitrag schon 1983 den Begriff der Dualisierung ${ }^{2}$ - sich nicht aufgrund individueller Defizite (fehlende Qualifikation, Deutschkenntnisse, Flexibilität) an den Rändern des Arbeitsmarktes wiederfinden - wie es in einem auch für Deutschland wesentlichen Strang der aktivierenden Arbeitsmarktpolitik vermutet wurde -, sondern als Ergebnis von Strategien von Arbeitgebern, Beschäftigtenvertretern und dem Staat auf der Suche nach einem Ausgleich für die Konflikte und Widersprüche am Arbeitsmarkt.

\section{III.}

So wie die Soziologie eine multiparadigmatische Wissenschaft ist, so ist es auch die Arbeitsmarktsoziologie. Doch aufgrund der prägenden Fragen, die von Segmentierung, Mobilität und Arbeitslosigkeit handeln, betonen die dominanten Ansätze eher Strukturen als Handeln, eher Interessen als Interaktionen, und eher die Systemintegration als die Sozialintegration. Dies sind auch die vorherrschenden Perspektiven von Claus Offe, wobei bei ihm der Fokus weniger auf einer wie auch immer gearteten Integration liegt, sondern eher auf Integrationsproblemen und Desintegrationserscheinungen aufgrund von Logiken der kapitalistischen Produktionsweise. $\mathrm{Zu}$ Recht ist die theoretische Grundeinstellung Claus Offes als neo-marxistisch und funktionalistisch beschrieben worden.

2 Offe, C. (2018): Perspektiven auf die Zukunft des Arbeitsmarktes. „Orthodoxie“, „Realismus“ und „dritte Wege“ (orig. 1983). In: Macht und Effizienz. Studien zur kapitalistischen Rationalisierung der Arbeit. Offe, C., Hrsg.; Wiesbaden: Springer Fachmedien Wiesbaden (Ausgewählte Schriften von Claus Offe), pp 43-59. 
Demnach sind die Produktionsverhältnisse und dort wiederum die Eigentumsverhältnisse zentral für das Verständnis von Wirtschaft und Gesellschaft. Sie bestimmen eine machtasymmetrische, hierarchische, von Interessen und Konflikten geprägte Sozialstruktur innerhalb einer Gesellschaft. Die Frage, ob sich diese Sozialstruktur im Kern auf zwei Klassen - Arbeiterklasse und Kapitalisten - zurückführen lässt, oder ob es innerhalb und außerhalb dieser Klassen weitere wesentliche Gruppen gibt (wie z. B. Angestellte, Selbstständige, Solo-Selbstständige, unterwertig und überwertig entlohnte Beschäftigte, hybrid Beschäftigte), markiert eine Linie, die den dogmatischen von einem potenziell gehaltvollen Marxismus trennt. Charakteristisch für den Neo-Marxismus ist die Thematisierung der Rolle des Staates: Er ist nicht bloßer Agent des Kapitals, sondern eher eine Arena widerstreitender Interessen, die in den Produktionsverhältnissen ihre Wurzel haben: „The structural arrangements of the welfare state tend to make people ignore or forget that the needs and contingencies that the welfare state responds to are themselves constituted, directly or indirectly, in the sphere of work and production, and that a reliable conception of social security does, therefore, presuppose not only the expansion of ,citizen rights“, but of „workers rights“ in the process of production“ (Some Contradictions of the Modern Welfare State, orig. 1981, hier abgedruckt in Bd. 2: 79-87; hier: 87). Zugleich ist er mehr als eine Arena, denn er ist auch eine Struktur, in der die Akteure agieren. Die Handlungsmöglichkeiten des Staates sind insbesondere dann eng begrenzt, wenn er sich auf sozial ausgleichende Reparaturen beschränken möchte, die gerade nicht bis in die „sphere of work and production“ eingreifen. Und mitunter sind pragmatisch-realistische Reparaturvorschläge, die Dysfunktionalitäten am Arbeitsmarkt beheben sollen, nicht einmal sozial ausgleichend, etwa wenn Arbeitslosigkeit dadurch bekämpft werden soll, dass Arbeitslose zu einer aktiveren Arbeitssuche und höheren Konzessionsbereitschaft angeregt werden sollen, wie Offe (gemeinsam mit Hinrichs) schon frühzeitig argumentierte. ${ }^{3}$

Der Funktionalismus zeigt sich bei Offe darin, dass eher Handlungslogiken als tatsächliches Handeln untersucht werden, und Handlungslogiken als gebunden an Interessen, Ressourcen und die Möglichkeiten ihrer Mobilisierung verstanden werden. Die Frage, wer eigentlich der Akteur ist, gerät darüber mitunter in den Hintergrund. Die ableitbaren Handlungsfolgen nehmen in den Analysen oft einen größeren Raum ein als das empirisch beobachtbare Handeln. Entsprechend

3 Und zwar in dem oben erwähnten Aufsatz von 1983. Mitten in der Diskussion um Arbeitsmarktreformen, die zu den Hartz-Reformen führten, sagte Offe - ein langjähriger Befürworter eines Grundeinkommens - aber auch: ,Wenn alle Bürger einen Anspruch auf eine minimale (steuer- statt beitragsfinanzierte) soziale Grundsicherung hätten, dann wäre die Forderung nach mehr Flexibilität moralisch überzeugender“ (Die Widersprüche des Sozialen, orig. 2001, Bd. 1: 153-159; hier: 158). 
sind die Schlussfolgerungen oft weitreichend, im Regelfall aber als Hypothesen zu begreifen, selbst wenn sie nicht als solche ausgeflaggt werden.

Die auf diese Weise von Claus Offe gewonnenen Ergebnisse (oder: Hypothesen) sind, soweit ich das empirisch beurteilen kann, teilweise widerlegt, teilweise bestätigt worden, auf jeden Fall anhaltend lesenswert. Vor allem aber ist festzustellen: Sie strukturieren konträre Positionen und schaffen mit theoretischen Mitteln Übersicht. Sie weisen zudem auf Probleme hin, die in empirischen Arbeiten zum Arbeitsmarkt nur sehr selten thematisiert werden, insbesondere die ungleichheitsverstärkende Komponente des Sozialstaates durch die Konditionalisierung sozialstaatlicher Leistungen, wenn die individuelle Existenzsicherung aufgrund fehlender oder unzureichender Erwerbsintegration gefährdet ist. Vor diesem Hintergrund ist es nicht überraschend, dass sich die wachsende Orientierung der Arbeitsmarktsoziologie an empirischen Daten in den Aufsätzen von Claus Offe kaum niedergeschlagen hat. Eher lässt sich aus den Arbeiten von Claus Offe ein anhaltender Impuls für empirische Forschung formulieren, nämlich Prozesse der Definition von Status, der sozialstaatlichen Zuweisung von Personen zu Statuskategorien und der individuellen Verarbeitung dieser Status zu untersuchen. Eine Stärke seiner Beiträge besteht darin, dass sie wiederholt konkurrierende Interpretationen eines Phänomens, etwa des Wachstums des Dienstleistungssektors oder gängige Empfehlungen, beispielsweise zum Umgang mit Strukturwandel und Arbeitslosigkeit, rekonstruieren und vergleichend analysieren. Was es bei Offe weniger gibt, zumindest soweit es seine Arbeiten zur Soziologie des Arbeitsmarktes betrifft, sind explizit theorievergleichende oder -rekonstruierende Aufsätze.

Dies ließe vermuten, dass es Offe auch darauf abgesehen hat, in politische Debatten einzugreifen. Hierzu gibt es einen Beitrag von 1975 (der älteste Aufsatz in dem sechsbändigen Werk überhaupt), in dem Offe Möglichkeiten und Grenzen der Politikberatung erörtert und darauf hinweist, dass Adressaten von Politikberatung keineswegs nur Regierungs- und Parteipolitiker:innen oder staatliche Verwaltung sein müssen, sondern etwa auch Akteure der Zivilgesellschaft sein können. Die in staatlichen Beratungsnachfragen von vornherein vorgenommenen, weder von beratungssuchenden Auftraggebern noch den hinzugezogenen Wissenschaftler:innen in Frage gestellten Beschränkungen der Fragestellungen auf einen engen Kreis des administrativ vielleicht schwierigen, politisch aber leicht konsensfähigen Bereich des Handelns, veranlasste Claus Offe zu dem Schluss, „dass die praktisch erfolgreichste Art der ,Politikberatung“ im Verzicht auf Beratung besteht und sich stattdessen einer empirisch-analytischen Politikforschung zuwendet, die den Akteuren des politisch-administrativen Systems präzise die für ihr Handeln konstitutive Realitätsverleugnung vor Augen führt; das könnte das bescheidene Ergebnis haben, das Festhalten an dieser Realitätsverleugnung zu 
erschweren“ (Die kritische Funktion der Sozialwissenschaften, orig. 1975, hier abgedruckt in Bd. 3: 379-386; hier: 386). Möglicherweise hat die etwa 30 Jahre später einsetzende und bis heute starke Nachfrage von Politik und Verwaltung nach daten- und evidenzbasierter Arbeitsförderung dazu beigetragen, dass Claus Offe verstärkt jenseits des Mainstreams der Arbeitsmarktforschung tätig war, die er zumindest in ihrer formativen Phase deutlich geprägt hat. Mag die Arbeitsmarktsoziologie in der Bundesrepublik kein hegemoniales Forschungsprogramm gehabt haben, so ist Claus Offe doch seinem Forschungsprogramm gefolgt, das sich als stabil erwiesen hat. Es hat seinen Kern in der Analyse von Funktionszusammenhängen im Bereich von Arbeit und Produktion, reicht aber über diesen Kern weit hinaus.

Danksagung: Ich danke Philipp Langer und Thorsten Schlee für die Diskussionen zur Vorbereitung dieses Beitrages. 